\title{
Comparative Nucleotide Sequence of Encoding for Quaking Protein of Birds and Mammals
}

\author{
Tomoaki Murata $^{*}, 1$, Yasuhiro Yamashiro ${ }^{2}$, Tatsuya Kondo ${ }^{3}$, Akiteru Amimoto ${ }^{4}$, Michinori Shirasu ${ }^{5}$, \\ Masahiro Yasuda ${ }^{6}$, Noboru Ito $^{7}$, Minako Hino ${ }^{8}$, Munekazu Nakaichi ${ }^{9}$, Satoshi Une ${ }^{9}$, \\ Kazuhito Itamoto ${ }^{9}$ and Yasuho Taura ${ }^{9}$
}

\author{
${ }^{1}$ Science Research Center, Yamaguchi University, 1-1-1 Minamikogushi, Ube City, Yamaguchi 755-8505, Japan \\ ${ }^{2}$ Faculty of Health Science, Graduate School of Medicine, Yamaguchi University, 1-1-1 Minamikogushi, Ube City, \\ Yamaguchi 755-8505, Japan \\ ${ }^{3}$ School of Medicine, Kumamoto University, 1-1-1 Honjo, Kumamoto City, Kumamoto 860-8556, Japan \\ ${ }^{4}$ Amica Pet Clinic, 3-2-3 Onda, Ube City, Yamaguchi 755-0023, Japan \\ ${ }^{5}$ Tokiwa Yuenkyokai, 3-4-1 Norisada, Ube City, Yamaguchi 755-0003, Japan \\ ${ }^{6}$ Faculty of Agriculture, Miyazaki University, 1-1 Gakuenkibanadainishi, Miyazaki City, Miyazaki 889-2192, Japan \\ ${ }^{7}$ School of Veterinary Medicine, Rakuno Gakuen University, 582 Bunkyodaimidorimachi, Ebetsu City, Hokkaido \\ 069-8501, Japan \\ ${ }^{8}$ Master of Health Science, Yamaguchi University, 1-1-1 Minamikogushi, Ube City, Yamaguchi 755-8505, Japan \\ ${ }^{9}$ Faculty of Agriculture, Yamaguchi University, 1677-1 Yoshida, Yamaguchi City, Yamaguchi 753-8511, Japan
}

\begin{abstract}
Partial nucleotide sequences of eight kinds of bird (common crane, gray pelican, goose, black swan, bewisk's swan, whooper swan, ostrich, and chicken) gene encoding quaking protein were analyzed. Variously sized bands were observed in all eight birds compared with the same sized band in mammals based on nested PCR results, and all sequence data showed a very high level of homology. Three bases (CD223(Ala)) in exon 6 of all eight bird species were defective compared with sequence data from the mouse, and both the existence and defect of 24 bases (CD211 219) in exon 6 of the eight birds were confirmed on complementary DNA sequence analysis. These findings suggest that birds generate specific alternative sequencing data, there are many alternative splicing variants, and the avian quaking gene may be a specific marker differentiating between birds and mammals.
\end{abstract}

The mouse quaking I ( $q k I)$ gene, encoding a $\mathrm{KH}$ domain containing RNA-binding protein [1], was first isolated as a candidate gene for neurological mutation, quaking viable, and was known as a model for human leukodystrophy [2]. Genes highly homologous to $q k I$ have been found in various animal species from nematodes to humans [3-7], and mutations in these genes result in various developmental defects other than demyelination. It is therefore believed that members of the $q k I$ gene family are essential for multiple biological processes. Herein, we report the isolation and sequence analysis of eight kinds of avian $q k$.

Genomic DNA was isolated from whole blood cells of eight kinds of bird (common crane, gray pelican, goose, black swan, bewisk's swan, whooper swan, ostrich, and chicken) by the standard method. RNA was isolated from whole blood cells of these birds with Trizol LS reagent (Invitrogen, USA). The ostrich was one-week-old and the others were adults, and none showed abnormality according to

*Address correspondence to this author at the Science Research Center, Yamaguchi University, 1-1-1 Minamikogushi, Ube City, Yamaguchi 7558505, Japan; Tel: +81 83622 2306; Fax: +81 83622 2412;

E-mail: ceb@yamaguchi-u.ac.jp neurological examinations. Complementary DNA synthesis was performed using the SuperScript Preamplification System (Invitrogen, USA). Primers used for the reverse transcription reaction were qk-5c (5'-CAAAGGCGATTACCAG TTAAC-3') and qk-5D (5'-GGTTAGATGGTAAGACGAA C-3'). The PCR primers were designed from highly conserved nucleotide sequences between mice and humans based on the results obtained in our previous study [3, 4]. Using these cDNA and gDNA as a template, PCR and nested PCR were performed for amplifications using the following primer sets: e2-4(5'-AGCTGCGGAGCCTGCAATAT-3'), EH5-3(5'-CTCCATCAGCTGCATCTTC-3'), qk-5c, qk-5d, EH5-5(5'-GAAGATGCAGCTGATGGAG-3'), and e6-u (5'-GCATGACAGCGGTCTGTATTT-3'). The PCR reactions were performed with the following cycling parameters: at $94^{\circ} \mathrm{C}$ for $4 \mathrm{~min}$, then 38 cycles comprising denaturation at $94^{\circ} \mathrm{C}$ for $30 \mathrm{~s}$, annealing at $60^{\circ} \mathrm{C}$ for $30 \mathrm{~s}$, and extension at $72^{\circ} \mathrm{C}$ for $60 \mathrm{~s}$. Nested PCR reactions were performed for 30 cycles. Amplified cDNA and gDNA products were directly sequenced using the Dye Terminator cycle sequencing kit with an ABI 373A DNA sequencer (Applied Biosystems). Primers used for sequence determination were EH5-5 and e6-u. 


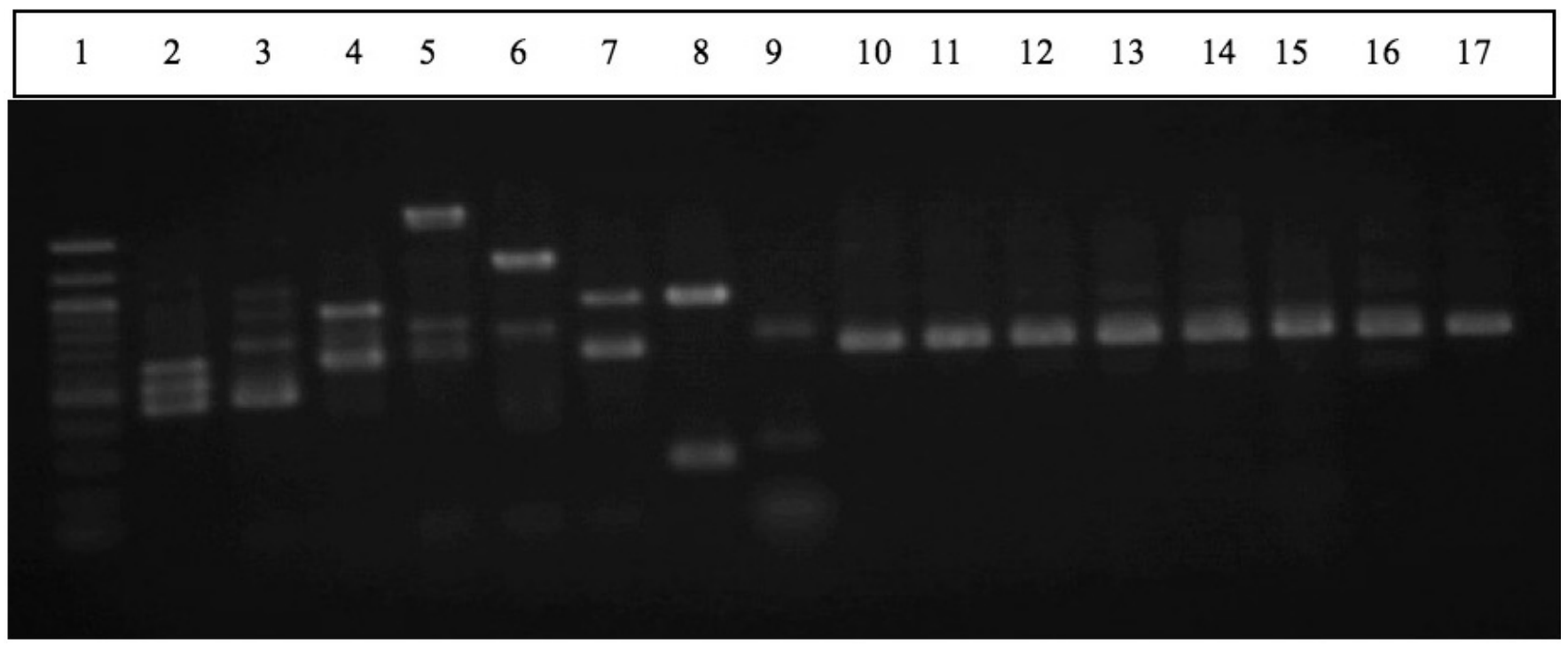

Fig. (1) Electrophoresis pattern for intron 5 - exon 6 of eight kinds of bird and eight kinds of mammal. primers: e2-4 \& EH5-3, 1.5\% TBE gel, 100V, 40min, lane 1: 100bp ladder marker; lane 2: common crane; lane 3: grey pelican; lane 4: goose; lane 5: black swan; lane 6: bewisk's swan; lane 7: whooper swan; lane 8: ostrich; lane 9: chicken; lane 10: mouse; lane 11: rat; lane 12: rabbit; lane 13: dog; lane 14: cat; lane 15: cow; lane 16: pig; lane 17: horse.

Electrophoresis patterns were detected (Fig. 1). Many variously sized bands were clearly confirmed in all eight kinds of bird, compared with one same-sized band and a very thin differently-sized band in eight kinds of mammal. Nucleotide sequences of these cDNA and gDNA quaking genes around intron 5 - exon 6 are shown in parallel with the $q k I$ sequence in Fig. (2). Nucleotide sequences of all avian $q k$ showed an absence of three bases (CD223 (Ala)) com- pared to $q k I$ and just one amino acid (Fig. 2). The alternative splicing (-24 bp (CD211 219: CA/GCC/CTT/GCC/TTT/ $\mathrm{TCT} / \mathrm{CTT} / \mathrm{GCA} / \mathrm{G})$ ) in exon 6 of all avian $q k$ was also confirmed.

The sequence of our chicken $q k I$ and that of Mezquita's chicken $q k I[2]$ are the same, but the source organs are different. This shows that the three bases defect and the twenty four base alternative splicing in exon 6 of $q k$ are normal and

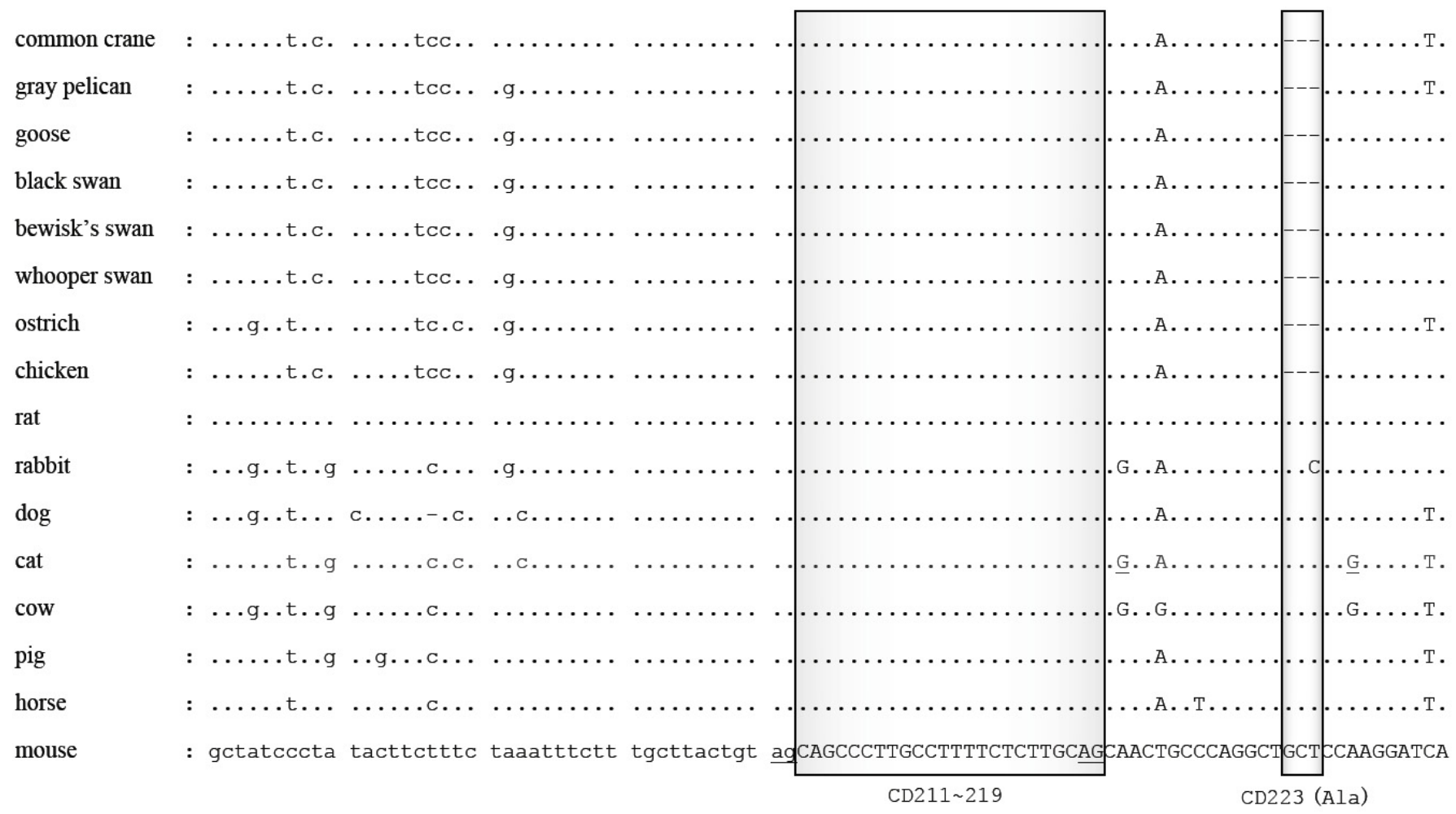

Fig. (2). Intron 5 - exon 6 of genomic DNA of whole blood cells of eight kinds of bird and eight kinds of mammal, (dot): same base with base of mouse, small letter: base in intron 5, large letter: base in exon 6, _(underline): splicing base, -: defected base. 
ubiquitous in the chicken. In addition, the structures of exon 6 of $q k$ were very highly conserved in all birds, but were not so highly conserved between birds and mammals. Fujita reported the alternative splicing patterns for $q k I$ in the rat brain [8]. This article suggests that splicing occurs in a very small portion, is usual in birds.

The evolutionary dichotomy between birds and mammals began more than three hundred million years ago. The highlevel homology of the quaking gene was confirmed in the same class of animals from our data, but there was a lower homology between different classes, Aves and Mammalia. This suggests that this $q k$ is a specific marker differentiating between birds and mammals.

The sequence of avian $q k$ showed that it easily underwent alternative splicing (Murata et al. personal data, preparation). In the mammals, alternative splicing occurred, but effective repair systems such as nuclease might be active to maintain protein conformity. In these birds, its activity may be weak, or the activity of spliceosome or legated enzyme may be strong. The spliceosome may be good analytical tool to investigate the development and differences between birds and mammals.

The sequence data reported in this paper were deposited in the DNA Data Bank of Japan (DDBJ) as follows: common crane: grgrqkI-01 AB284384, gray pelican: pecrqkI-01 AB2 84385, goose: ananqkI-01 AB284386, black swan: cyatqkI-
01 AB284387, bewisk's swan: cycoqkI-01 AB284388, whooper swan: cycyqkI-01 AB284389, ostrich: stcaqkI-01 AB284 391, and chicken: gagaqkI-01 AB284390.

\section{REFERENCES}

[1] Ebersole TA, Chen Q, Justice MJ, Artzt K. The quaking gene product necessary in embryogenesis and myelination combines features of RNA binding and signal transduction proteins. Nat Genet 1996; 12: 260-5.

[2] Mezquita J, Pau M, Mezquita C. Four isoforms of the signaltransduction and RNA-binding protein QKI expressed during chicken spermatogenesis. Mol Reprod Dev 1998; 50: 70-8.

[3] Murata T, YamashiroY, Kondo T, Une S, Nakaichi M. Nucleotide sequence of cDNA for canine and feline quaking protein. J Jpn Vet Med Assoc 2002; 55: 505-8.

[4] Murata T, YamashiroY, Kondo T, Nakaichi M, Une S, Taura Y. Nucleotide sequence of complementary DNA encoding for quaking protein of cow, horse and pig. DNA Seq 2005; 16: 300-3.

[5] Tanaka H, Abe K, Kim C. Cloning and expression of the quaking gene in the zebrafish embryo. Mech Dev 1997; 69: 209-13.

[6] Zaffran S, Astier M, Gratecos D, Semeriva M. The held out wing (how) drosophila gene encodes a putitive RNA-binding protein involved in the control of muscular and cardiac activity. Development 1997; 124: 2087-98.

[7] Zorn AM, Grow M, Patterson KD, et al. Remarkable sequence conservation of transcripts encoding amphibian and mammalian homologous of quaking a $\mathrm{KH}$ domain RNA-binding protein. Gene 1997; 188: 199-206.

[8] Fujita N, Sato S, Kurihara T, et al. cDNA cloning of mouse myelin-associated glycoprotein: A novel alternative splicing pattern. Biochem Biophys Res Commun 2004; 165: 1162-9. 